\title{
Sinonasal Adenocarcinoma-Experience of an Oncology Center
}

\author{
Teresa Bernardo, Edite Ferreira, Joaquim Castro Silva, Eurico Monteiro \\ Serviço de Otorrinolaringologia, Instituto Português de Oncologia do Porto, Porto, Portugal \\ Email: mtefebe@gmail.com
}

Received December 1, 2012; received December 29, 2012; accepted January 8, 2013

\begin{abstract}
Introduction and Objectives: Sinonasal tumours represent only $3 \%$ of all head and neck cancers. Adenocarcinoma is the second most frequent histopathology type. Hardwood exposure has been considered a risk factor. Sinonasal adenocarcinoma grows silently which leads to a late diagnosis and low survival rates. The aim of this study was to present our experience in the management of the patients with sinonasal adenocarcinoma. Method: Retrospective medical records review of patients with sinonasal adenocarcinomas (1974 to 2009). Results: From 301 patients with sinonasal tumors, 67 had histology of adenocarcinoma. Patient average age was $60.1 \pm 11.1$ years (30 - 84 years). $83.6 \%$ were man. $65.7 \%$ had history of working with wood. $70.1 \%$ of the patients had advance disease. The most common treatment strategy was external surgery (lateral rhinotomy $(47.8 \%)$, sublabial $(17.9 \%)$ or cranio-facial resection $(6 \%)$ ) or endoscopic approaches with postoperative radiotherapy. The 3 and 5 years overall survival rate were $60 \%$ and $49 \%$, respectively. Conclusions: Our group study showed similar epidemiologic characteristics than other series. We confirmed sinonasal adenocarcinomas tendency to late diagnosis and wood dust exposure relation. In our experience, the limited surgical treatment (without craniofacial resection) and postoperative radiotherapy has good survival rates results, similar to other departments who consider the craniofacial resection as the standard treatment.
\end{abstract}

Keywords: Sinonasal Adenocarcinomas; Wood Dust; Surgical Treatment; Radiotherapy

\section{Introduction}

Sinonasal tumors are rare forms of head and neck malignant neoplasms accounting for only $3 \%$ of head and neck cancers $[1,2]$. They represent less than $1 \%$ of all tumours with an annual European incidence of $1-2 / 100,000$ inhabitants $[2,3]$. Furthermore, adenocarcinoma accounts for only a small percentage $(10 \%-20 \%)$ of all sinonasal cancers [4], although it is the second most frequent histopathology type after squamous cell carcinoma $[5,6]$. The average age for presentation is 50 - 60 years, with male predominance $(2$ - 4:1) [4,7]. Epidemiologically, adenocarcinoma has been associated with workers exposed to hardwood dust since this relation was first reported in 1965 by MacBeth $[1,8]$. These are mostly intestinal-type sinonasal adenocarcinomas and show a strong preference for the ethmoid sinus [4]. Some authors supported that cigarette smoking acts in a synergic manner $[2,5]$.

Sinonasal adenocarcinoma grows silently with no symptoms, which leads to a late diagnosis and low survival rates. Nodes and distant metastases are rare at presentation. Patient survival depends on local control that is extremely difficult because of the anatomical proximity of the orbit and brain [3]. Local recurrence is frequent.
Craniofacial resection (CFR) has been adopted worldwide as the standard treatment modality for tumours of the paranasal sinuses involving the anterior skull base [1] For other histological types of tumors like esthesioneuroblastoma, CFR offers a better prognosis than other forms of treatment [1]. Unfortunately, for adenocarcinoma of the ethmoid sinus, CFR has not rendered significant improvement in survival rates [1]. Lateral rhinotomy followed by radiotherapy has recently been shown to produce comparable results [1]. This said we would like to question the real need for using CFR. This is pertinent since CFR involves a lot of co-morbidities and does not imply a significant increase in the survival rates.

The aim of this study is to present our experience in the management of patients with sinonasal adenocarcinomas from the North of Portugal. We highlight the epidemiology characteristics, the risk factors, the treatment strategies and the survival rates.

\section{Methods}

After approval by the institutional review board, a search of the ENT Department and sinus cancer database was performed to identify all patients who underwent treat- 
ment for sinonasal cancer between January 1974 and December 2009 at our tertiary comprehensive cancer care center.

Medical records from the patients who had been diagnosed and treated for primary sinonasal adenocarcinoma were reviewed for information regarding demographics, diseases characteristics, location and extent of the tumour, stage, histopathology findings, treatment strategies and oncologic outcomes.

Tumours localization was harvested from these medical records. Disease was re-staged in accordance to the American Joint Committee on Cancer (AJCC) TNM staging, 7th Edition, 2010.

Treatment strategies included surgery, radiotherapy and chemotherapy separately or in combination. The surgical approaches were partial or total maxillectomy or sphenoethmoidectomy by endonasal, sublabial or lateral rhinotomy approaches. Orbit exentration and CFR was done when there was periorbit and dural significant invasion, respectively.

Radiotherapy was given by doses of $1.8 \mathrm{~Gy} /$ day, 5 days a week in a total dose of $60-65$ Gy. The technique most often used combines one anterior and two lateral fields of $\mathrm{Co}^{60}$ gamma or $6 \mathrm{MV}$ photons X. Until 2002 the irradiation isodoses were determined by conventional dosimetry techniques. Since then, computed tomography three dimensional dosimetry has been used.

The chemotherapy regimen most used was a combination of Cisplatin and 5-Fluorouracil.

Descriptive statistics for frequencies of study patients within the category for each of the parameters of interest were enumerated with software assistance. Curves describing overall and disease-specific survival rate were generated by the Kaplan-Meier product limit method. The statistical significance of differences between the actuarial curves was tested by the log-rank test. A p value less than 0.05 were considered significant.

\section{Results}

After the From 301 patients with sinonasal tumors, 67 (22.3\%) had a positive histology for adenocarcinoma with an average follow-up time of 45.25 months (2 - 204 months). That was the second most frequent histopathology after squamous cell carcinoma (155\% - 51.5\%). Patient average age was $60.1 \pm 11.1$ years $(30-84$ years $)$. Fifty-six patients $(83.6 \%)$ were man, $44(65.7 \%)$ had history of working with wood and 24 (35.8\%) had smoke habits. For survival rates calculations we excluded 5 patients that received no treatment, 2 that died before treatment was started and other 3 that received only palliative therapy due to very advanced disease or/and poor general condition.

Fifty-one patients (76.1\%) had only nasal symptoms at presentation and 5 had nasal symptoms with another lo- cation symptom. The nasal symptoms were mainly nasal obstruction with or without unilateral secretions and/or nose bleeding. The mean time before presenting symptoms was 7.2 months (1 - 24 months).

The tumour most frequent localization was the ethmoid sinus (73\%). Intestinal type adenocarcinoma $(28 \%$ - 41.8\%) was the second most frequent histopathology after adenocarninoma with no other specification. Twentythree patients $(34.3 \%)$ had tumor extension to orbit (9), skull base (2), orbit and skull base (8) and others places like pterigopalatine fossa and cheek (5). According to $\mathrm{T}$ stages, 1 (1.5\%) patient was T1, $19(28.4 \%) \mathrm{T} 2,21$ (31.3\%) T3, 21 (31.3\%) T4a and 5 (7.5\%) T4b. Two patients were $\mathrm{N}+(\mathrm{N} 1$ and $\mathrm{N} 3)$ and only one patient was M1 on presentation. Finally, $20(29.9 \%)$ patients were at an early stage (Stage I and II) and the others 47 (70.1\%) had advance disease (Stage III, IVa and IVb) (Table 1).

The most common treatment strategy was surgery with postoperative radiotherapy (48 patients: $71.6 \%$ ). The other patients were mostly submitted to surgery alone $(12 \%-17.9 \%)$, one patient received pre-operative radiotherapy and other pre-operative chemoradiotherapy.

Forty-seven $(70.1 \%)$ patients were submitted to an external surgical approach: lateral rhinotomy $(32 \%-47.8 \%)$, sublabial $(12 \%-17.9 \%)$ and CFR $(4 \%-6 \%)-13$ patients were treated by endoscopic surgery and 2 patients do not had surgical approach information. Seven patients $(10.4 \%)$ were submitted to orbit exenteration.

The 2, 3 and 5 years overall survival rates were $70 \%$, $60 \%$ and $49 \%$, respectively (Figure 1). It was worst in most advanced disease stages $(\mathrm{p}<0.05)$ and better after 2002 ( $p>0.05)$ but there were more patients at early

Table 1. Patients stage distribution.

\begin{tabular}{cc}
\hline \multicolumn{2}{c}{ Patients Stage Distribution Number (\%) } \\
\hline Early (I and II) & $20(29.9 \%)$ \\
Advanced (III and IV) & $47(70.1 \%)$ \\
\hline
\end{tabular}

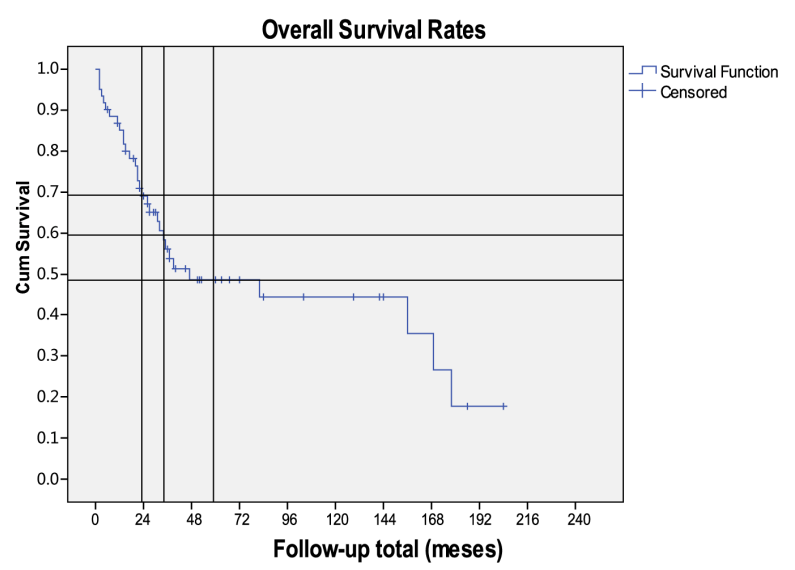

Figure 1. Kaplan-Meier curve for overall survival in sinonasal adenocarcinomas. 
stages since then $(\mathrm{p}<0.05)$.

The 2, 3, 5 and 10 years disease-specific survival rates were $75 \%, 69 \%, 69 \%$ and 49\%, respectively (Figure 2). There was no difference between patients treated before and after 2002 but it was better when higher radiotherapy doses were used ( $\geq 60 \mathrm{~Gy})$ ( $p>0.05$ ).

There was no significant statistical difference on overall and disease-specific survival rate between the groups that received and did not received radiotherapy but the last group had more patients with early stage disease.

Of the 62 patients that received any treatment, $34.3 \%$ experienced recurrence with an average disease-free time of 35.25 months. This recurrence was local in $69.6 \%$.

\section{Discussion}

Our group study showed similar epidemiology characteristics than other series $[1,7,9]$. We found an average age of 60 years and a male predominance. Most of our patients were wood workers. These suggest a causal relation like in others studies $[2,8,9]$. The ethmoid localization and the histopathology subtype support it [4]. Almost $40 \%$ of the patients had smoking habits that make us suspect that it may be a risk factor.

We confirmed these tumour growths with no symptoms and the tendency for advanced stages at presentation. This fact reduces the choice of treatment and approach to be used [7] and explain the higher recurrence/ persistence rates and decreased survival.

The treatment strategies were mostly surgery and postoperative radiotherapy. We performed orbital exenteration (7) and CFR (4) when there was significant periorbit and dural invasion, respectively. As opposed to other series $[7,10]$, we do not support low threshold for performing CFR because we argue that the high rate of surgical complication and morbidity overpass the survival rates improvements.

The introduction of CFR made possible the treatment

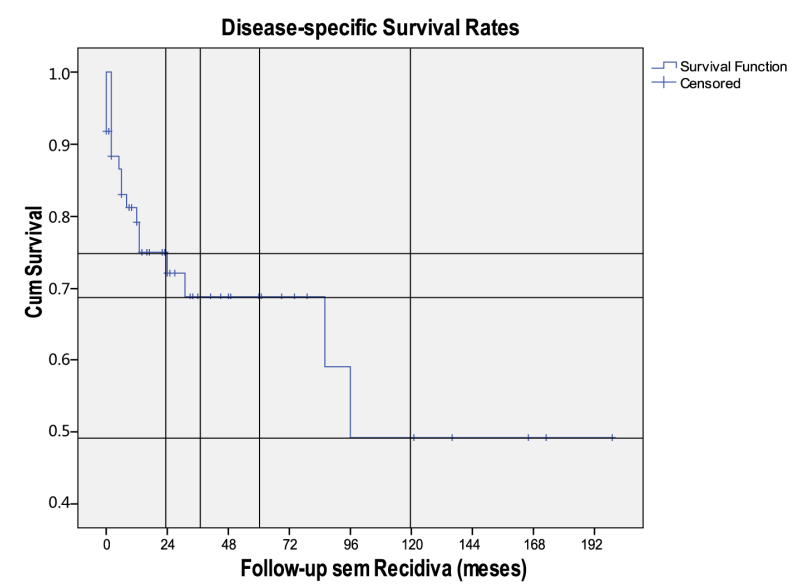

Figure 2. Kaplan-Meier curve for specific survival in sinonasal adenocarcinomas. of more advanced diseases, previously considered inoperable, and even consideration of salvage surgery in recurrences. When the anterior fossa is involved, the results in terms of complications and survival rate are considerably worse [7]. In ethmoid adenocarcinoma, despite the improvements in surgical and anesthetic techniques, survival data with CFR approach remain disappointing [1]. Our survival rates results confirm this suspicion with no great difference to other studies that currently perform CFR, whose overall survival rates at 5 and 10 years goes from $36 \%$ to $57 \%$ and $28 \%$ to $53 \%$, respectively [7,11-15].

Our good survival results also suggest that for well selected cases and with the appropriate use of adjuvant therapy, endoscopic resection of sinonasal cancer results in acceptable oncologic outcomes.

Complementary radiotherapy is advisable in all cases, except in small, well-defined tumours, where surgical treatment seems to be sufficient. It is important as adenocarcinoma complementary treatment because of the possibility of existence of tumoral islands in healthy mucosa at areas far from the tumour and retropharyngeal metastases [7]. We did not notice a significant difference between the patients submitted and not submitted to radiotherapy because the last group had a greater number of patients with earlier disease. This could also mean that the postoperative radiotherapy in advanced cases had a positive role. There was better overall survival but no specific-disease survival results after 2002, when we started to use computed tomography three dimensional dosimetry. That means the disease control is the same but the morbidity is provably inferior. Three-dimensional computed tomography-based dosimetry allowed for better mapping of the target volume and preservation of surrounding structures [16]. Of course we have to take into account that patients after 2002 had earlier diagnosis and that could be the real cause for better morbidity.

We only used pre-operative radiotherapy in two patients. Some authors support there is no clear difference in the sequence of surgery and radiotherapy in the management of sinonasal cancer patients [17].

\section{Conclusion}

Sinonasal adenocarcinomas are rare tumors with aggressive biological behaviour and no specific presentations symptoms. Wood dust exposure is a well known risk factor. In our experience, the limited surgical treatment (with no CFR) and postoperative radiotherapy has good survival rates results, similar to other departments which have a low threshold for CFR and consider it as the standard treatment.

\section{REFERENCES}

[1] P. P Knegt, K. W. Ah-See, L. A. Velden and J. Kerrebijn, "Adenocarcinoma of the Ethmoidal Sinus Complex: Sur- 
gical Debulking and Topical Fluorouracil May Be the Optimal Treatment," Archives of Otolaryngology_Head \& Neck Surgery, Vol. 127, No. 2, 2001, pp. 141-146. doi: $10.1080 / 02841860902874755$

[2] B. A. McMonagle and M. Gleeson, "Nasal Cavity and Paranasal Sinus Malignancy," In: M. G. Scott-Brown, Otorhinolaryngology, Head and Neck Surgery, 7th Edition, Edward Arnold Publishers Ltd., Londres, 2008, pp. 24172436.

[3] G. Gatta, G. Bimbi, L. Ciccolallo, G. Zigon, G. Cantú and EUROCARE Working Group, "Survival for Ethmoid Sinus Adenocarcinoma in European Populations," Acta Oncologica, Vol. 48, No. 7, 2009, pp. 992-998.

[4] I. Leivo, "Update on Sinonasal Adenocarcinoma: Classification and Advances in Immunophenotype and Molecular Genetic Make-Up," Head and Neck Pathology, Vol. 1, No. 1, 2007, pp. 38-43. doi:10.1007/s12105-007-0025-2

[5] J. H. Kuijpens, M. W. Louwman, R. Peters, G. O. Janssens, A. L. Burdorf and J. W. Coebergh, "Trends in Sinonasal Cancer in the Netherlands: More Squamous Cell Cancer, Less Adenocarcinoma: A Population-Based Study 1973-2009," European Journal of Cancer, Vol. 48, No. 15, 2012, pp. 2369-2374.

[6] J. H. Turner and D. D. Reh, "Incidence and Survival in Patients with Sinonasal Cancer: A Historical Analysis of Population-Based Data," Head and Neck, Vol. 34, No. 6, 2012, pp. 877-885. doi:10.1002/hed.21830

[7] J. L. Llorente, F. Núñez, J. P. Rodrigo, R. Fernández León, C. Alvarez, M. Hermsen, et al., "[Sinonasal AdenoCar-Cinomas: Our Experience]," Acta Otorrinolaringológica Española, Vol. 59, No. 5, 2008, pp. 235-238.

[8] A. d'Errico, S. Pasian, A. Baratti, R. Zanelli, S. Alfonzo, L. Gilardi, et al., "A Case-Control Study on Occupational Risk Factors for Sino-Nasal Cancer," Occupational and Environmental Medicine, Vol. 66, No. 7, 2009, pp. 448455. doi:10.1136/oem.2008.041277

[9] R. Breheret, L. Laccourreye, C. Jeufroy and A. Bizon, "Adenocarcinoma of the Ethmoid Sinus: Retrospective Study of 42 Cases," European Annals of Otorhinolaryngology, Head and Neck Diseases, Vol. 128, No. 5, 2011, pp. 211-217. doi:10.1016/j.anorl.2011.02.012
[10] E. Hanna, F. DeMonte, S. Ibrahim, D. Roberts, N. Levine and M. Kupferman, "Endoscopic Resection of Sinonasal Cancers with and without Craniotomy: Oncologic Results," Archives of Otolaryngology - Head \& Neck Surgery, Vol. 135, No. 12, 2009, pp. 1219-1224. doi:10.1001/archoto.2009.173

[11] V. J. Lund, D. J. Howard, W. I. Wei and A. D. Cheesman, "Craniofacial Resection for Tumor of the Nasal Cavity and Paranasal Sinuses: A 17-Year Experience," Head and Neck, Vol. 20, No. 2, 1998, pp. 97-105. doi:10.1002/(SICI)1097-0347(199803)20:2<97::AID-HE D1>3.0.CO;2-Y

[12] J. P. Shah, D. H. Kraus, M. H. Bilsky, P. H. Gutin, L. H. Harrison and E. W. Strong, "Craniofacial Resection for Malignant Tumors Involving the Anterior Skull Base," Archives of Otolaryngology — Head \& Neck Surgery, Vol. 123, No. 12, 1997, pp. 1312-1317. doi:10.1001/archotol.1997.01900120062010

[13] D. Salvan, M. Julieron, P. Marandas, F. Janot, A. M. Leridant, C. Domenge, et al., "Combined Transfacial and Neurosurgical Approach to Malignant Tumors of the Ethmoid Sinus," The Journal of Laryngology \& Otology, Vol. 112, No. 5, 1998, pp. 446-450. doi: $10.1017 / \mathrm{S} 0022215100140745$

[14] G. Cantu, C. L. Solero, R. Miceli, F. Mattana, S. Riccio, S. Colombo, et al., "Anterior Craniofacial Resection for Malignant Paranasal Tumors: A Monoinstitutional Experience of 366 Cases," Head and Neck, Vol. 34, No. 1, 2012, pp. 78-87. doi:10.1002/hed.21685

[15] I. Ganly, S. G. Patel, B. Singh, D. H. Kraus, P. G. Bridger, G. Cantu, et al., "Craniofacial Resection for Malignant Paranasal Sinus Tumors: Report of an International Collaborative Study," Head and Neck, Vol. 27, No. 7, 2005, pp. 575-584. doi:10.1002/hed.20165

[16] Y. Chu, H. G. Liu and Z. K. Yu, "Patterns and Incidence of Sinonasal Malignancy with Orbital Invasion," Chinese Medical Journal (English Edition), Vol. 125, No. 9, 2012, pp. 1638-1642.

[17] R. H. Jesse, "Preoperative versus Postoperative Radiation in the Treatment of Squamous Carcinoma of the Nasal Sinus," The American Journal of Surgery, Vol. 110, No. 4, 1965, pp. 552-556. doi:10.1016/0002-9610(65)90036-X 